\title{
Quality of Life Assessment in Pediatric Epilepsy
}

\author{
Sujana Shakya ${ }^{1}$ Rajani Shakya ${ }^{1}$ Prithuja Poudel ${ }^{2}$ Ashwinee Kumar Shrestha ${ }^{1}$ \\ ${ }^{1}$ Department of Pharmacy, Kathmandu University, Dhulikhel, Nepal \\ 2Dhulikhel Hospital, Kathmandu University Teaching Hospital, \\ Dhulikhel, Nepal \\ Address for correspondence Rajani Shakya, PhD, Department \\ of Pharmacy, Kathmandu University, Dhulikhel, Nepal \\ (e-mail: rajani@ku.edu.np).
}

Int J of Ep:2020;6:54-58

\begin{abstract}
Objectives Quality of life (QOL) is an accepted health outcome measure in clinical practice. This study was performed to assess QOL in pediatric epilepsy patients and identify demographic and clinical factors influencing it.

Materials and Methods A cross-sectional study was conducted in the outpatient pediatric department of Dhulikhel Hospital, a tertiary care teaching hospital in Nepal. Parents or caretakers of children receiving one or more antiepileptic drugs were interviewed using $\mathrm{QOL}$ childhood epilepsy questionnaire comprising 91 items with seven domains and 16 subscales.

All the individual subscales of the questionnaire were found to be compromised with least score on QOL and general health item. Compromised social, physical, psychological, emotional, cognitive as well as behavioral domains were also identified.

Results QOL was found to be more impaired in older children and in those from rural areas. They had significant linguistic impairment and lower level of self-esteem. Overall QOL was found to be compromised more in patients with generalized than with focal epilepsy. Seizure

Keywords

- epilepsy

- quality of life

- pediatrics frequency and polytherapy were the most important factors influencing overall QOL.

Conclusion Despite taking appropriate antiepileptic drugs, epilepsy was found to be detrimental to QOL of patients. Hence, $\mathrm{QOL}$ assessment should be an important part of epilepsy management protocol.
\end{abstract}

\section{Introduction}

Epilepsy is a chronic illness that is still considered a social taboo in developing countries such as Nepal. Epilepsy, due to its chronicity, puts pediatric patients at risk of poor quality of life $(\mathrm{QOL}) .^{1} \mathrm{QOL}$ is defined by the World Health Organization (WHO) as the individual's perception of their position in life, in the context of the culture and value systems in which they live and in relation to their goals, expectations, standards, and concerns. QOL assessment provides a broad concept of the impact of epilepsy and antiepileptic medications in pediatric patients. ${ }^{2,3}$ Healthcare professionals can make informed decisions regarding medication, side effects, and the overall well-being by assessing QOL of pediatric patients with epilepsy. Several epilepsy-specific QOL scales have been developed to measure accurately the impact and burden of epilepsy. However, their target populations, details of the origin of the items, and psychometric properties vary significantly. ${ }^{3}$

This study was conducted to assess parent-reported QOL in pediatric patients with epilepsy and identify the demographic and clinical factors influencing it. This is the first study of its kind in Nepal to use epilepsy-specific instrument for assessing the influence of demographic and clinical variables on QOL of pediatric patients with epilepsy.

\section{Materials and Methods}

A cross-sectional study was conducted in the pediatric outpatient department (OPD) of Dhulikhel Hospital, a tertiary care teaching hospital located $\sim 30 \mathrm{~km}$ away from Kathmandu, the capital city of Nepal. Patients were diagnosed to have epilepsy on the basis of clinical history and electroencephalography
DOI https://doi.org/ 10.1055/s-0040-1718787 ISSN 2213-6320. (c) 2020. Indian Epilepsy Society.

This is an open access article published by Thieme under the terms of the Creative Commons Attribution-NonDerivative-NonCommercial-License, permitting copying and reproduction so long as the original work is given appropriate credit. Contents may not be used for commercial purposes, or adapted, remixed, transformed or built upon. (https://creativecommons.org/licenses/by-nc-nd/4.0/)

Thieme Medical and Scientific Publishers Pvt. Ltd., A-12, 2nd Floor, Sector 2, Noida-201301 UP, India 
changes. Consecutive pediatric patients with stable seizure frequency attending the OPD clinic for follow-up were enrolled in the study. All patients were receiving antiepileptic medications. Patients with chronic medical condition (asthma, hypertension, chronic renal failure, chronic lung disease, thalassemia, hypothyroidism, etc.) were excluded. Patients whose primary caregivers were not available to answer the questionnaire and those who were considered for alternate therapies including dietary therapy or surgery were also excluded.

Two documents incorporating the study variables were required to be completed; quality of life childhood epilepsy 1 (QOLCE-1) for collecting information on demographic and clinical variables, and QOLCE-2 to evaluate the QOL of the pediatric patients with epilepsy. The QOLCE-2 is a parental, multifaceted epilepsy-specific scale developed by Sabaz et al for the measurement of QOL in pediatric patients with epilepsy. ${ }^{4}$ Parent form consists of 91 questions with 16 subscales covering 7 domains: QOL domain, physical activities, well-being, cognition, social activities, behavior, and general health. Permission was obtained from the author for its use along with instructions for coding and questionnaire. Questionnaire was translated into local language by bilingual language expert and again translated back to the English language by another language expert. Comparison of the data obtained from translated and back translated questionnaire was performed. Negligible differences were rectified after comparison. Reliability of the translated questionnaire was established using the Cronbach's $\alpha$ value. Ethical approval from the Institutional Review Board of the hospital was obtained. Written informed consent was taken from the caregiver of the patient to participate in the study. The QOLCE questionnaire was administered in a uniform manner and by a single investigator for all the study subjects.

The effect on individual subscales was evaluated from the scoring provided by the author. Each QOLCE question has a rating scale with qualitative headings (e.g., "excellent," "very good," "good," "fair," and "poor"). To statistically analyze these rating scales, quantitative values were allocated. The five types of rating were linearly converted to a 100-point scale where zero was the lowest or poorest score and hundred reflected the highest level of functioning. The higher the score, the better is the $\mathrm{QOL}$ of the pediatric patients. The subscale score was calculated by computing the mean of the items belonging to each subscale. An overall QOL score was computed by adding each subscale score for each individual and then dividing by 16 .

Statistical analysis was performed using SPSS version 20. Demographic and clinical variables were expressed in terms of numbers and proportions and QOL scores were expressed as mean and standard deviation. The mean QOL scores were compared among demographic and clinical variables using parametric tests such as Student's $t$-test and analysis of variance (ANOVA). The individual subscale score was also calculated according to author's instructions and the overall QOL was calculated. The individual subscales were compared among demographic and clinical variables using ANOVA. A $p$-value $<0.05$ was considered statistically significant.

\section{Results}

The questionnaire converted into local Nepali language was utilized that was tested for its internal consistency and reliability using Cronbach's $\alpha$. The Cronbach's $\alpha$ value for each individual subscale was greater than 0.7.

A total of 62 pediatric patients participated in the study. The demographic characteristics of pediatric patients and their relationship with the QOL scores are shown in - Table 1. Demographic variables found to be significantly associated with QOL were age group and residence.

QOL was found to be better in younger patients when compared with the older age groups, and this difference was statistically significant $(p<0.05)$. The QOL was also found to be significantly affected by the area of residence of the participants $(p<0.05)$. Residence in rural areas was associated with lower QOL values. In this study, majority of the patients were from the urban area (72.6\%). Majority of the patients in the study were male (56.5\%). Education of mothers of the children with epilepsy was evaluated. Majority of them were found to be illiterate. However, it did not affect the QOL of children.

The epilepsy-related and treatment-related variables of pediatric patients and their relationship with the QOL scores are shown in -Tables 2 and 3, respectively. The variables significantly associated with QOL were type of epilepsy, seizure frequency in the last 1 year, and number of antiepileptic drugs.

In this study, the most common type of epilepsy observed was generalized epilepsy (53.2\%). Though QOL was found

Table 1 Relationship of demographic variables with QOL

\begin{tabular}{|c|c|c|c|}
\hline Variable & $\begin{array}{l}\text { Number of } \\
\text { participants, } \\
n(\%)\end{array}$ & $\begin{array}{l}\text { Mean } \\
\text { QOL } \pm \text { SD }\end{array}$ & $p$-Value \\
\hline \multicolumn{4}{|l|}{ Age group } \\
\hline $0-4$ & $13(21)$ & $64.09 \pm 12.47$ & \multirow[t]{4}{*}{$0.01^{\mathrm{a}}$} \\
\hline $5-9$ & 21 (33.9) & $54.95 \pm 8.49$ & \\
\hline $10-14$ & $19(30.6)$ & $50.01 \pm 17.50$ & \\
\hline $15-19$ & $9(14.5)$ & $46.76 \pm 11.15$ & \\
\hline \multicolumn{4}{|l|}{ Residence } \\
\hline Rural & $17(27.4)$ & $46.21 \pm 14.66$ & \multirow[t]{2}{*}{$0.005^{\mathrm{a}}$} \\
\hline Urban & $45(72.6)$ & $57.17 \pm 12.62$ & \\
\hline \multicolumn{4}{|l|}{ Gender } \\
\hline Male & $35(56.5)$ & $53.08 \pm 13.87$ & \multirow[t]{2}{*}{0.491} \\
\hline Female & $27(43.5)$ & $55.57 \pm 14.27$ & \\
\hline \multicolumn{4}{|l|}{ Maternal education } \\
\hline Illiterate & $27(43.5)$ & $49.91 \pm 11.63$ & \multirow[t]{3}{*}{0.088} \\
\hline Primary school & $21(33.9)$ & $54.18 \pm 15.60$ & \\
\hline High school & $14(22.6)$ & $60.51 \pm 12.25$ & \\
\hline
\end{tabular}

Abbreviations: QOL, quality of life; SD, standard deviation. ${ }^{\text {a }} p$-Value is significant. 
Table 2 Relationship of epilepsy related variables with QOL

\begin{tabular}{|c|c|c|c|}
\hline Variable & $\begin{array}{l}\text { Number of } \\
\text { participants, } \\
n(\%)\end{array}$ & $\begin{array}{l}\text { Mean QOL } \pm \\
\text { SD }\end{array}$ & $p$-Value \\
\hline \multicolumn{4}{|l|}{ Type of epilepsy } \\
\hline Generalized & $33(53.2)$ & $50.70 \pm 14.08$ & \multirow[t]{2}{*}{$0.036^{\mathrm{a}}$} \\
\hline Focal & $29(46.8)$ & $58.11+13.01$ & \\
\hline \multicolumn{4}{|c|}{ Seizure frequency in last $1 \mathrm{y}$} \\
\hline Weekly & $3(4.8)$ & $30.65 \pm 12.70$ & \multirow[t]{5}{*}{$<0.001$} \\
\hline Monthly & $8(12.9)$ & $40.05 \pm 11.20$ & \\
\hline 6 monthly & $11(17.7)$ & $52.25 \pm 14.09$ & \\
\hline Yearly & $4(6.5)$ & $54.47 \pm 13.36$ & \\
\hline Nil since last year & $36(58.1)$ & $59.81 \pm 10.37$ & \\
\hline
\end{tabular}

Abbreviations: QOL, quality of life; SD, standard deviation. ${ }^{a} p$-Value is significant.

Table 3 Relationship of treatment related variables with QOL

\begin{tabular}{|l|l|l|l|}
\hline Variable & $\begin{array}{l}\text { Number of } \\
\text { participants, } \\
n(\%)\end{array}$ & $\begin{array}{l}\text { Mean } \\
\text { QOL } \pm \text { SD }\end{array}$ & -Value \\
\hline Antiepileptic drugs & \multicolumn{2}{|l|}{$0.025^{a}$} \\
\hline Monotherapy & $48(77.4)$ & $56.30 \pm 12.06$ & \\
\hline Polytherapy & $14(22.6)$ & $46.85 \pm 17.82$ &
\end{tabular}

Abbreviations: QOL, quality of life; SD, standard deviation. ${ }^{a} p$-Value is significant.

to be affected by both generalized and focal epilepsy, the overall QOL was more significantly compromised in patients with generalized epilepsy as indicated by the lower values of mean QOL for focal epilepsy. QOL was significantly affected by seizure frequency. Greater the frequency of seizures since last 1 year, more affected was the QOL.

Majority of the pediatric patients enrolled in the study were on monotherapy (77.4\%). The number of antiepileptic medications used had a significant effect on QOL. Patients on polytherapy had lower QOL than those on monotherapy.

Among the 16 QOL subscales, "quality of life item" subscale and "general health item" subscale were severely affected as shown in - Table 4 . The subscale of anxiety was least affected. All other subscales also had a compromised score. Overall QOL of pediatric patients suffering from epilepsy was highly compromised as indicated by a score of $54.16 \%$.

The effects of different demographic and epilepsyrelated variables were evaluated on individual subscales as shown in - Table 5. Among the variables, age group, residence, type of epilepsy, and seizure frequency were significantly associated with QOL. Therefore, their effects on individual subscales were evaluated. The $p$-values were evaluated for significant differences to assess how they particularly affected the individual subscales. Age group was found to affect 7 out of 16 subscales, residence was shown to affect 11 out of 16 subscales, type of seizure was shown to affect 7 out of 16 subscales, and seizure frequency was shown to affect 12 out of 16 subscales.
Table 4 Scores for individual subscales

\begin{tabular}{|l|l|l|}
\hline Serial no. & Subscales & Score \\
\hline 1 & QOL item & 29.43 \\
\hline 2 & Physical restrictions & 41.5 \\
\hline 3 & Energy/fatigue & 49.6 \\
\hline 4 & Depression & 71.3 \\
\hline 5 & Anxiety & 78.8 \\
\hline 6 & Control/helplessness & 61.1 \\
\hline 7 & Self-esteem & 58.7 \\
\hline 8 & Attention/concentration & 51.6 \\
\hline 9 & Memory & 62.6 \\
\hline 10 & Language & 61.7 \\
\hline 11 & Other cognitive & 51.1 \\
\hline 12 & Social interaction & 52.4 \\
\hline 13 & Social activities & 51.9 \\
\hline 14 & Stigma item & 60.5 \\
\hline 15 & Behavior & 55.0 \\
\hline 16 & General health item & 29.4 \\
\hline & Overall QOL & 54.16 \\
\hline
\end{tabular}

Abbreviation: QOL, quality of life.

\section{Discussion}

Measures to assess the health-related $\mathrm{QOL}$ of pediatric patients with epilepsy are lacking in Nepal. In this study, the questionnaire converted into local Nepali language was utilized that was tested for its internal consistency and reliability using Cronbach's $\alpha$. According to Tavakol and Dennick, calculating $\alpha$ is a common practice in medical education research when multiple-item measure of a concept or construct is employed. ${ }^{5}$ The value for individual subscales was greater than 0.7 which is the accepted criterion.

It was found that demographic variables such as age and residence and clinical variables such as type of epilepsy, seizure frequency in the last 1 year and the number of antiepileptic medications were the major predictors significantly affecting QOL in pediatric patients with epilepsy. ${ }^{6}$ Overall QOL was affected more in older pediatric patients and those living in rural areas. Similarly, pediatric patients with generalized epilepsy, higher seizure frequency, and those receiving polytherapy had more compromised overall QOL.

Age of pediatric patients with epilepsy was observed to affect the overall QOL. Age affected seven subscales significantly. Overall QOL was more affected in older than in younger age group that was similar to that reported by Devinsky et al. ${ }^{7}$ Younger patients scored better in terms of physical restrictions and energy/fatigue. Similarly, emotional and cognitive domains were also better in younger age groups. According to Nadkarni et al, this might be because older pediatric patients are more likely to perceive greater negative impact on life and have more negative attitude toward epilepsy. ${ }^{8}$ Language and memory were also affected in the older age group. Caplan et al had also demonstrated that older children had more language impairment and a wider range of linguistic defects. ${ }^{9}$ 
Table 5 Individual subscales and significance of demographic and epilepsy-related variables

\begin{tabular}{|c|c|c|c|c|c|}
\hline \multirow[t]{2}{*}{ Serial no. } & \multirow[t]{2}{*}{ Subscales } & \multicolumn{4}{|c|}{$p$-Value } \\
\hline & & Age group & Residence & $\begin{array}{l}\text { Type of } \\
\text { epilepsy }\end{array}$ & Seizure frequency \\
\hline 1 & Quality of life item & 0.624 & $0.001^{\mathrm{a}}$ & 0.188 & $0.021^{\mathrm{a}}$ \\
\hline 2 & Physical restrictions & $0.004^{\mathrm{a}}$ & $0.012^{\mathrm{a}}$ & $0.015^{\mathrm{a}}$ & $0.002^{\mathrm{a}}$ \\
\hline 3 & Energy/fatigue & $0.042^{\mathrm{a}}$ & $0.010^{\mathrm{a}}$ & 0.263 & $0.001^{\mathrm{a}}$ \\
\hline 4 & Depression & 0.055 & 0.276 & 0.447 & $0.003^{\mathrm{a}}$ \\
\hline 5 & Anxiety & 0.437 & 0.455 & 0.788 & 0.058 \\
\hline 6 & Control/helplessness & $0.006^{\mathrm{a}}$ & 0.073 & $0.010^{\mathrm{a}}$ & 0.105 \\
\hline 7 & Self-esteem & $0.013^{\mathrm{a}}$ & $0.004^{\mathrm{a}}$ & $0.005^{\mathrm{a}}$ & $0.000^{\mathrm{a}}$ \\
\hline 8 & Attention/concentration & $0.015^{\mathrm{a}}$ & $0.004^{\mathrm{a}}$ & $0.038^{\mathrm{a}}$ & $0.002 a$ \\
\hline 9 & Memory & 0.100 & $0.015^{\mathrm{a}}$ & $0.024^{\mathrm{a}}$ & $0.001^{\mathrm{a}}$ \\
\hline 10 & Language & $0.026^{\mathrm{a}}$ & $0.012^{\mathrm{a}}$ & $0.040^{\mathrm{a}}$ & $0.001^{\mathrm{a}}$ \\
\hline 11 & Other cognitive & $0.006^{\mathrm{a}}$ & $0.03^{a}$ & $0.023^{\mathrm{a}}$ & $0.004^{\mathrm{a}}$ \\
\hline 12 & Social interaction & 0.185 & $0.018^{\mathrm{a}}$ & 0.120 & $0.004^{\mathrm{a}}$ \\
\hline 13 & Social activities & 0.921 & 0.146 & 0.308 & 0.572 \\
\hline 14 & Stigma item & 0.228 & 0.168 & 0.160 & 0.068 \\
\hline 15 & Behavior & 0.118 & $0.009^{a}$ & 0.747 & $0.006^{\mathrm{a}}$ \\
\hline 16 & General health item & 0.157 & $0.015^{\mathrm{a}}$ & 0.449 & $0.000^{\mathrm{a}}$ \\
\hline
\end{tabular}

${ }^{a} p$-Value is significant.

This may be due to easier adaptability and greater learning skills in younger age groups as indicated by Aggarwal et al. ${ }^{10}$ The lower self-esteem in older age group may be because of the fact that older children may feel ashamed of their condition and worry about social isolation. This fact also might have restricted them in terms of physical activities and energy.

QOL in patients living in rural areas was compromised to a greater extent when compared with those living in urban areas. QOL item and general health item might have decreased due to greater difficulty in getting access to the medical facility and the negative perception of people related to the disease in rural areas. The low literacy levels in rural areas might have led to lower social, cognitive, and behavioral scores as well as diminished self-esteem. Pal et al also studied significant social deficit in children with epilepsy residing in the rural areas. ${ }^{11}$ Overall, QOL was more compromised in patients with generalized epilepsy than those with focal epilepsy. Generalized epilepsy begins from both hemispheres of the brain compared with focal epilepsy that begins only from one of the hemispheres. Patients with generalized epilepsy not only have more severe seizures but also have more social problems, both at home and school, due to which children lose their self-esteem. Generalized epilepsy leads to severe impairment of physical, social, and emotional functioning and finally to general deterioration of one's QOL. Generalized epilepsy, in this study, affected seven subscales significantly including physical restrictions, control/helplessness, self-esteem, attention/concentration, memory, language, and cognitive subscales.

Greater the seizure frequency, lesser was the QOL. Greater seizure frequency in the last 1 year severely compromised the QOL in pediatric patients with epilepsy. Sabaz et al proved that QOLCE is sensitive to differences in seizure severity. ${ }^{4}$ Greater seizure frequency significantly affected 12 out of 16 subscales. Patients on polytherapy were found to have lower QOL than those on monotherapy that might be due to the reason that multiple medications were provided only to patients whose seizures were not controlled by monotherapy. ${ }^{8}$

Using an epilepsy-specific questionnaire, QOLCE, the overall QOL was obtained from mean of individual subscales that was found to be 54.16 on a total score of 100 . The overall QOL was found to be highly compromised. The individual subscales also have a decreased score according to 100-point item scale with compromise in social, physical, psychological, emotional, cognitive as well as behavioral domains, which suggests that treatment of epilepsy is not only about seizure control. There is a need for an integrated healthcare approach that should also include their QOL.

\section{Conclusion}

The overall QOL was found to be compromised in children with epilepsy. A periodic follow-up of their QOL is desirable and appropriate interventions should be performed to improve this factor. QOL assessment should be an essential part of the treatment protocol of epilepsy.

\section{Conflict of Interest}

S.S. reports grants from University Grants Commission (UGC), Nepal, during the conduct of the study. Rest authors declare no conflict of interest. 


\section{References}

1 Leonardi M, Ustun TB. The global burden of epilepsy. Epilepsia 2002;43(Suppl 6) :21-25

2 World Health Organization, International Classification of Functioning, Disability, and Health. Geneva: WHO; 2001

3 Ronen GM, Streiner DL, Rosenbaum P. Health-related quality of life in childhood epilepsy: moving beyond 'seizure control with minimal adverse effects'. Health Qual Life Outcomes 2003; $1(1): 36$

4 Sabaz M, Cairns DR, Lawson JA, Nheu N, Bleasel AF, Bye AME. Validation of a new quality of life measure for children with epilepsy. Epilepsia 2000;41(6):765-774

5 Tavakol M, Dennick R. Making sense of Cronbach's alpha. Int J Med Educ 2011;2:53-55
6 Gambhir SK, Kumar V, Singhi PD, Goel RC. Public awareness, understanding \& attitudes toward epilepsy. Indian J Med Res 1995;102:34-38

7 Devinsky O, Westbrook L, Cramer J, Glassman M, Perrine K, Camfield C. Risk factors for poor health-related quality of life in adolescents with epilepsy. Epilepsia 1999;40(12):1715-1720

8 Nadkarni J, Jain A, Dwivedi R. Quality of life in children with epilepsy. Ann Indian Acad Neurol 2011;14(4):279-282

9 Caplan R, Siddarth P, Vona P, et al. Language in pediatric epilepsy. Epilepsia 2009;50(11):2397-2407

10 Aggarwal A, Datta V, Thakur LC. Quality of life in children with epilepsy. Indian Pediatr 2011;48(11):893-896

11 Pal DK, Chaudhury G, Sengupta S, Das T. Social integration of children with epilepsy in rural India. Soc Sci Med 2002; 54(12):1867-1874 